\title{
Exactitud diagnóstica de la Escala de Depresión Posnatal de Edimburgo: consecuencias del tamizaje en mujeres mexicanas
}

\author{
Emma del C. Macías-Cortés, ${ }^{1 *}$ Virgilio Lima-Gómez ${ }^{1}$ y Juan Asbun-Bojalir
}

'Secretaría de Salud, Hospital Juárez de México; 'Instituto Politécnico Nacional, Escuela Superior de Medicina, Sección de Estudios de Posgrado. Ciudad de México, México

\section{Resumen}

Introducción: La depresión posparto es un episodio depresivo no psicótico con repercusiones graves en el vínculo de la madre con su hijo, de ahí la importancia de detectarla oportunamente. Objetivo: Determinar la exactitud de la Escala de Depresión Posnatal de Edimburgo como prueba diagnóstica y analizar las consecuencias del tamizaje y la probabilidad de depresión después de aplicar la prueba. Método: Tamizaje con la Escala de Depresión Posnatal de Edimburgo a 411 mujeres durante el posparto; se utilizó el Inventario de Depresión de Beck como referencia. Resultados: En un punto de corte de 12 , con la Escala de Depresión Posnatal de Edimburgo se obtuvo sensibilidad de $70.4 \%$, especificidad de $72.2 \%$, valor predictivo positivo de $36.9 \%$ y valor predictivo negativo de $91.4 \%$, así como un valor del área bajo la curva de 0.729 y $p=0.0003$. De 49 mujeres sin atención para depresión posparto, en cinco se identificó que la necesitaban. Conclusiones: La Escala de Depresión Posnatal de Edimburgo tiene una exactitud moderada; su aplicación es sencilla, accesible y debería ser rutinaria. Es necesario que en México se implementen estrategias para detectar y tratar la depresión posparto.

PALABRAS CLAVE: Depresión posparto. Escala de Depresión Posnatal de Edimburgo. Sensibilidad. Especificidad. Tamizaje. Exactitud diagnóstica.

\section{Diagnostic accuracy of the Edinburgh Postnatal Depression Scale: consequences of screening in Mexican women}

\begin{abstract}
Introduction: Postpartum depression is a non-psychotic depressive episode with serious repercussions on the bond between the mother and her child, hence the importance of detecting it in a timely manner. Objective: To determine the accuracy of the Edinburgh Postnatal Depression Scale as a diagnostic test and to analyze the consequences of screening and the probability of depression after applying the test. Method: Screening of 411 women with the Edinburgh Postnatal Depression Scale during the postpartum period; Beck's Depression Inventory was used as reference. Results: At a cutoff point of 12, a sensitivity of $70.4 \%$, specificity of $72.2 \%$, positive predictive value of $36.9 \%$ and negative predictive value of $91.4 \%$ were obtained with Edinburgh Postnatal Depression Scale, as well as an area under the curve of 0.729 and a p-value of 0.0003 . Out of 49 women without treatment for postpartum depression, five were identified to require it. Conclusions: The Edinburgh Postnatal Depression Scale has moderate accuracy; its application is simple, accessible and should be routine. It is necessary for strategies to detect and treat postpartum depression to be implemented in Mexico.
\end{abstract}

KEY WORDS: Postpartum depression. Edinburgh Postnatal Depression Scale. Sensitivity. Specificity. Screening. Diagnostic accuracy.

Correspondencia:

*Emma del C. Macías-Cortés

E-mail: ecmc2008@ hotmail.es
Fecha de recepción: 25-07-2019

Fecha de aceptación: 16-12-2019

DOI: 10.24875/GMM.19005424
Gac Med Mex. 2020;156:202-208

Disponible en PubMed

www.gacetamedicademexico.com

0016-3813/๑ 2020 Academia Nacional de Medicina de México, A.C. Publicado por Permanyer. Este es un artículo open access bajo la licencia CC BY-NC-ND (http://creativecommons.org/licenses/by-nc-nd/4.0/). 


\section{Introducción}

La depresión posparto (DPP) es un episodio depresivo no psicótico leve a moderado que inicia después del parto y se extiende a lo largo del primer año posparto. ${ }^{1}$ En el mundo, su prevalencia es de 10 a $20 \%$ y en México, de 13 a $24 \%{ }^{5}$ Generalmente se resuelve espontáneamente en tres a seis meses, aunque en $25 \%$ de los casos puede durar más de un año. ${ }^{6}$ Es importante diagnosticar y tratar la DPP por el impacto emocional profundo en el vínculo de la madre con el hijo; se ha observado que los hijos de madres deprimidas tienen tres veces más riesgo de tener problemas emocionales y conductuales. ${ }^{7-10}$

Es fundamental que el personal de salud tome conciencia de la importancia de identificar la DPP con pruebas rápidas, económicas y sencillas. En 2016, Place et al. afirmaron que existen escasas publicaciones sobre prevención, detección y tratamiento de la DPP en México. La DPP se menciona en diversas políticas de salud, pero no se han implementado protocolos para detectarla y tratarla rutinariamente en las unidades obstétricas. ${ }^{11}$ Además, aunque se apliquen pruebas de tamizaje, no siempre se utilizan los resultados para el seguimiento y tratamiento; solo de 23 a $60 \%$ de las mujeres con prueba de rastreo positiva sigue un tratamiento adecuado..$^{12-14}$

La prueba más utilizada, recomendada y validada internacionalmente para el tamizaje de la DPP es la Escala de Depresión Posnatal de Edimburgo (EPDS); el Inventario de Depresión de Beck (IDB) es una escala validada internacionalmente para determinar la severidad de la depresión..$^{15,16}$ Los estudios sobre la validez de la EPDS como prueba diagnóstica señalan diferencias en los valores de sensibilidad, especificidad, valores predictivos, tipo de población, tamaño de muestra, puntos de corte e instrumentos diagnósticos utilizados como referencia. ${ }^{17}$ En México existen pocos reportes sobre el tamizaje con la EPDS. Alvarado et al. recomiendan un punto de corte de 11/12 (sensibilidad de $75 \%$ y especificidad de $93 \%$ ) a las cuatro semanas posparto. ${ }^{18}$ Sin embargo, no se han publicado informes sobre las consecuencias de estos resultados en la práctica clínica, ni un análisis del rendimiento y exactitud diagnóstica de la EPDS.

El objetivo principal de esta investigación fue determinar la exactitud de la EPDS como prueba diagnóstica, para lo cual se utilizó el IDB como método de referencia. Se calculó sensibilidad, especificidad, valores predictivos, curva ROC e índice de Youden en diferentes puntos de corte y se analizaron las consecuencias del tamizaje y la probabilidad de DPP después de la aplicación de la prueba.

\section{Método}

Estudio observacional, transversal, descriptivo, realizado en la consulta externa del Hospital Juárez de México, Secretaría de Salud, de febrero a noviembre de 2009, registrado con el número HJM1411/07.10.09.

Se incluyeron mujeres de 15 a 45 años, con escolaridad mínima de quinto año de primaria, cuyo parto hubiera sucedido de 15 días a ocho semanas antes de la aplicación de la EPDS y que concedieron verbalmente su consentimiento previa información de los objetivos y procedimientos de la investigación. Se excluyeron las mujeres con enfermedad tiroidea conocida y aquellas cuyos hijos presentaron malformaciones congénitas o enfermedad que requería atención médica. Las mujeres que cubrieron los criterios de selección contestaron el cuestionario de la EPDS, el cual consiste en 10 preguntas que exploran el estado de ánimo de la mujer en los últimos siete días, cada pregunta tiene una puntuación de 0 a 3 (máximo 30 puntos en total); se consideró que una puntuación $\geq 10$ era indicativa de DPP. Posteriormente, a quienes obtuvieron una puntuación $\geq 10$ en la EPDS se les solicitó responder las preguntas del IDB, el cual es un cuestionario autoaplicado de 21 preguntas cuya puntuación de 0 a 63 indica la intensidad de la depresión: de 5 a 9 puntos, mínima; de 10 a 18 puntos, depresión leve a moderada; de 19 a 29 puntos, moderada a severa; de 30 a 63 puntos, severa.

Se obtuvieron frecuencias y porcentajes de las características demográficas, así como medidas de tendencia central y de dispersión de las puntuaciones de las escalas. Se calcularon los valores de prevalencia, sensibilidad, especificidad, valores predictivos y razones de verosimilitud ( $\mathrm{RV})$, likelihoods ratios o probabilidades posprueba, con intervalos de confianza de $95 \%$ (IC $95 \%$ ), para lo cual se utilizó el programa MedCalc versión 19.0.3. En el IDB se tomó como referencia una puntuación de 10.

La sensibilidad se definió como el porcentaje de individuos con la enfermedad que presentan una prueba positiva y como especificidad, el porcentaje de individuos sin la enfermedad que presentan una prueba negativa. El valor predictivo positivo (VPP) es la probabilidad de que un paciente con un resultado positivo (anormal) presente la enfermedad; el valor predictivo negativo (VPN) es la probabilidad de no tener la enfermedad cuando el resultado de la prueba 
Tabla 1. Características demográficas de las mujeres con sospecha de depresión posparto, que obtuvieron $\geq 10$ puntos en la Escala de Depresión Posnatal de Edimburgo

\begin{tabular}{|l|c|c|c|c|}
\hline Estado civil & Escolaridad & Ocupación & Partos & Adicciones \\
\hline Casadas & Primaria & Hogar & Uno & No \\
\hline $44.8 \%(n=37)$ & $12.7 \%(n=10)$ & $89.9 \%(n=71)$ & $44.3 \%(n=35)$ & $91.1 \%(n=72)$ \\
\hline Unión libre & Secundaria & Trabajan fuera del hogar & Dos & Sí tabaco \\
\hline $40 \%(n=32)$ & $60.8 \%(n=48)$ & $7.6 \%(n=6)$ & $30.4 \%(n=24)$ & $3.8 \%(n=3)$ \\
\hline Solteras & Medio superior & Estudiantes & Sí alcohol \\
\hline $12.7 \%(n=10)$ & $5.1 \%(n=4)$ & $2.5 \%(n=2)$ & $15.2 \%(n=12)$ & $3.8 \%(n=3)$ \\
\hline Viudas/divorciadas & Licenciatura & & Cuatro & Sídrogas \\
\hline $0 \%(n=0)$ & $0 \%(n=0)$ & $3.8 \%(3)$ & $3.8 \%(3)$
\end{tabular}

es negativo (normal)..$^{19}$ De acuerdo con lo reportado en la literatura, para el cálculo de los valores predictivos se consideró una prevalencia (probabilidad previa) de DPP de $18.7 \%$.

Las RV indican qué tanto el resultado de una prueba diagnóstica incrementará o disminuirá la probabilidad preprueba. Se consideraron los siguientes criterios: ${ }^{20}$

- Mayor que 10 o menor que 0.1 , cambios significativos.

- 5-10 o 0.1-0.2, cambios moderados.

- 2-5 o 0.5-0.2, pequeños cambios.

- 1-2 y $0.5-1$, cambios muy pequeños y raramente importantes.

Para determinar la probabilidad de clasificar correctamente a un par de mujeres, una sana y otra enferma seleccionadas al azar, con los resultados de sus pruebas se graficó la curva ROC. Se consideraron mujeres con DPP aquellas con una puntuación $\geq 10$ en el IDB. Se reportó el área bajo la curva con IC $95 \%$ y se consideró que 0.5-0.7 indicaba baja exactitud; 0.7-0.9, moderada exactitud; $>0.9$, exactitud alta. ${ }^{21}$ Finalmente se calculó el índice de Youden, para valorar el rendimiento de la EPDS como prueba diagnóstica (valor de 1 indica prueba perfecta y valor de 0 , prueba inútil).

\section{Resultados}

Se aplicó la EPDS a 441 mujeres; $17.9 \%(n=79)$ obtuvo una puntuación $\geq 10$. En la Tabla 1 se describen las características sociodemográficas. El promedio de edad fue de $26 \pm 7$ años, en la mayoría se trataba de mujeres casadas (44.8\%), primigestas $(44.3 \%)$, con educación secundaria $(60.8 \%)$ y dedicadas exclusivamente al hogar (89.9\%). El promedio de días después del parto en el que se encontró positividad de la prueba fue de $28 \pm 13$.

El promedio de la puntuación de la EPDS fue de $5.7 \pm 5 ; 82.1 \%(n=362)$ obtuvo menos de 10 puntos.
Tabla 2. Severidad de la depresión posparto en mujeres con $\geq 10$ puntos en la Escala de Depresión Posnatal de Edimburgo

\begin{tabular}{|l|c|c|l|}
\hline \multirow{2}{*}{ Puntuación del IDB } & \multicolumn{2}{|c|}{ Frecuencia $(\mathbf{n}=\mathbf{7 9})$} & Severidad de la DPP \\
\cline { 2 - 3 } & $\%$ & $n$ & \\
\hline $0-9$ & 22.8 & 18 & Mínima \\
\hline $10-18$ & 39.2 & 31 & Leve a moderada \\
\hline $19-28$ & 24 & 19 & Moderada a severa \\
\hline $29-63$ & 13.9 & 11 & Severa \\
\hline
\end{tabular}

Se utilizó el Inventario de Depresión de Beck como método de referencia.

Las 79 mujeres con una puntuación $\geq 10$ tuvieron un promedio de $14.58 \pm 4.08$ puntos; en ese grupo, el promedio de puntuación en el IDB fue de $17 \pm 11.1$. A pesar de tener una puntuación $\geq 10$ en la EPDS, $22.8 \%$ (18/79) no presentó datos compatibles con DPP. La DPP leve a moderada fue la más frecuente (39.2\%), seguida de la moderada a severa (24\%) y solo $13.9 \%$ tuvo datos de DPP severa (Tabla 2 ).

En la Tabla 3 se muestra cómo se modificó la validez y la seguridad de la EPDS según diferentes puntos de corte:

- Sensibilidad: de $93.4 \%$ con un punto de corte $>10$ disminuyó a $70.4 \%$ con un punto de corte $>12$ (IC $95 \%$ = 84.1-98.2 y 57.4-81.5, respectivamente).

- Especificidad: de $16.6 \%$ con un punto de corte de 10 aumentó a $94.4 \%$ cuando el punto de corte fue 17; la especificidad fue de $72.2 \%$ cuando el punto de corte fue $>12$ (IC $95 \%=3.6-41.4$, 72.7-99.9 y 46.5-90.3, respectivamente).

- VPP: de $20.5 \%$ cuando la puntuación fue $>10$ se incrementó a $36.9 \%$ con un punto de corte $>12$ (IC $95 \%=17.2-24.3$ y $21.5-55.6$, respectivamente). 
Tabla 3. Valores de validez, seguridad y razones de verosimilitud, conforme el punto de corte de la Escala Posnatal de Edimburgo y el Inventario de Depresión de Beck como método de referencia

\begin{tabular}{|c|c|c|c|c|c|c|}
\hline $\begin{array}{l}\text { Punto de } \\
\text { corte EPDS }\end{array}$ & $\begin{array}{l}\text { Sensibilidad } \\
\% \text { (IC } 95 \% \text { ) }\end{array}$ & $\begin{array}{l}\text { Especificidad \% } \\
\text { (IC } 95 \%)\end{array}$ & $\begin{array}{c}\text { Razón de } \\
\text { verosimilitud } \\
\text { positivo (IC } 95 \% \text { ) }\end{array}$ & $\begin{array}{c}\text { Razón de } \\
\text { verosimilitud } \\
\text { negativo (IC } 95 \% \text { ) }\end{array}$ & $\begin{array}{c}\text { VPP } \\
\%(\text { IC } 95 \%)\end{array}$ & $\begin{array}{c}\text { VPN } \\
\%(\text { (IC 95) }\end{array}$ \\
\hline$\geq 10$ & $100(94.1-100)$ & $0.00(0.0-18.5)$ & $1.0(1.0-1.0)$ & - & $18.7(18.7-18.7)$ & - \\
\hline$>10$ & $93.4(84.1-98.2)$ & $16.6(3.6-41.4)$ & $1.1(0.9-1.4)$ & $0.3(0.1-1.6)$ & $20.5(17.2-24.3)$ & $91.7(73.1-97.8)$ \\
\hline$>11$ & $80.3(68.2-89.4)$ & $38.8(17.3-64.3)$ & $1.3(0.9-1.9)$ & $0.5(0.2-1.1)$ & $23.2(17.0-30.8)$ & 89.6 (79.9-94.9) \\
\hline$>12$ & 70.4 (57.4-81.5) & $72.2(46.5-90.3)$ & $2.5(1.2-5.4)$ & $0.4(0.3-0.7)$ & $36.9(21.4-55.6)$ & $91.4(86.8-94.5)$ \\
\hline$>13$ & $57.3(44.1-70)$ & $83.3(58.6-96.4)$ & $3.4(1.2-9.9)$ & $0.5(0.4-0.7)$ & $44.2(21.6-69.5)$ & $89.5(85.6-92.4)$ \\
\hline$>14$ & $47.5(34.6-60.7)$ & $83.3(58.6-96.4)$ & $2.8(1.0-8.3)$ & $0.6(0.5-0.9)$ & $39.6(18.4-65.6)$ & $87.4(83.4-90.5)$ \\
\hline$>15$ & $37.7(25.6-51)$ & $88.8(65.3-98.6)$ & $3.3(0.9-13.0)$ & $0.7(0.5-0.9)$ & $43.8(16.9-75.0)$ & $86.1(82.8-88.9)$ \\
\hline$>16$ & $31.1(19.9-44.3)$ & $88.8(65.3-98.6)$ & $2.8(0.7-10.9)$ & $0.7(0.6-1.0)$ & $39.2(14.2-71.5)$ & $84.9(81.6-87.7)$ \\
\hline$>17$ & $24.5(14.5-37.3)$ & 94.4 (72.7-99.9) & $4.4(0.6-31.3)$ & $0.8(0.7-1.0)$ & $50.4(12.6-87.8)$ & $84.5(81.9-86.7)$ \\
\hline$>18$ & $21.3(11.9-33.7)$ & $100(81.5-100)$ & - & $0.7(0.7-0.9)$ & 100 & $84.7(82.9-86.3)$ \\
\hline$>19$ & $13.1(5.8-24.2)$ & $100(81.5-100)$ & - & $0.8(0.8-1.0)$ & 100 & $83.3(81.9-84.7)$ \\
\hline$>20$ & $11.4(4.7-22.2)$ & $100(81.5-100)$ & - & $0.8(0.8-1.0)$ & 100 & $83.1(81.8-84.3)$ \\
\hline$>21$ & $9.8(3.7-20.2)$ & $100(81.5-100)$ & - & $0.9(0.8-1.0)$ & 100 & $82.8(81.6-84.0)$ \\
\hline$>22$ & $6.5(1.8-15.9)$ & $100(81.5-100)$ & - & $0.9(0.9-1.0)$ & 100 & $82.3(81.3-83.3)$ \\
\hline$>23$ & $4.9(1.0-13.7)$ & $100(81.5-100)$ & - & $0.9(0.9-1.0)$ & 100 & $82.1(81.2-82.9)$ \\
\hline$>26$ & $1.6(0.04-8.8)$ & $100(81.5-100)$ & - & $0.9(1.0-1.0)$ & 100 & $81.5(81.1-82.0)$ \\
\hline$>29$ & $0.0(0.0-5.9)$ & $100(81.5-100)$ & - & $1.0(1.0-1.0)$ & - & $81.3(81.3-81.3)$ \\
\hline
\end{tabular}

VPP = valor predictivo positivo, VPN = valor predictivo negativo, IC $95 \%$ = intervalo de confianza a $95 \%$

- VPN: de $91.7 \%$ cuando la puntuación fue $<10$ pasó a $91.4 \%$ cuando fue $<12$ (IC $95 \%=73.1$ 97.8 y $86.8-94.5$, respectivamente).

Considerando un punto de corte de 12 en la EPDS y de 10 en el IDB, la RV positiva fue de 2.5 y la RV negativa de 0.4 (IC $95 \%=1.2-5.4$ y $0.3-0.7$, respectivamente), que representan un cambio pequeño, pero significativo.

En la Figura 1 se muestran las consecuencias de realizar el tamizaje con la EPDS y en la Figura 2, la curva ROC: área bajo la curva $=0.729$ (IC $95 \%=$ 0.617-0.823), $p=0.0003$, con un error estándar de 0.0634 . El índice de Youden fue de 0.4271 y se sugirió el punto de corte en 12.

\section{Discusión}

El presente estudio es el primero que evalúa el rendimiento de la EPDS como prueba diagnóstica en mujeres mexicanas cuyo resultado del tamizaje fue positivo para DPP. La frecuencia de positividad en la EPDS (17.9 \%) concuerda con los resultados publicados previamente ${ }^{2-4}$ con diferentes puntos de corte, de 9 a 12 dependiendo del estudio.

Se encontró positividad de la EPDS a los $28 \pm 13.2$ días posparto, lo que sugiere la conveniencia de realizar rutinariamente el tamizaje a partir de la segunda semana del parto, cuando las mujeres acudan a revisión del recién nacido. Si bien nuestro objetivo no fue estudiar factores de riesgo de DPP, es importante resaltar que su detección aportaría información útil para implementar estrategias de prevención y tratamiento.

El Hospital Juárez de México atiende población de escasos recursos sin seguridad social, que probablemente tiene factores de riesgo como violencia intrafamiliar; de acuerdo con el Instituto Nacional de Estadística y Geografía, $43.9 \%$ de las mujeres mexicanas ha tenido al menos un episodio de violencia por parte de su pareja. ${ }^{22}$ México necesita implementar políticas de salud nacionales para cuidar el 


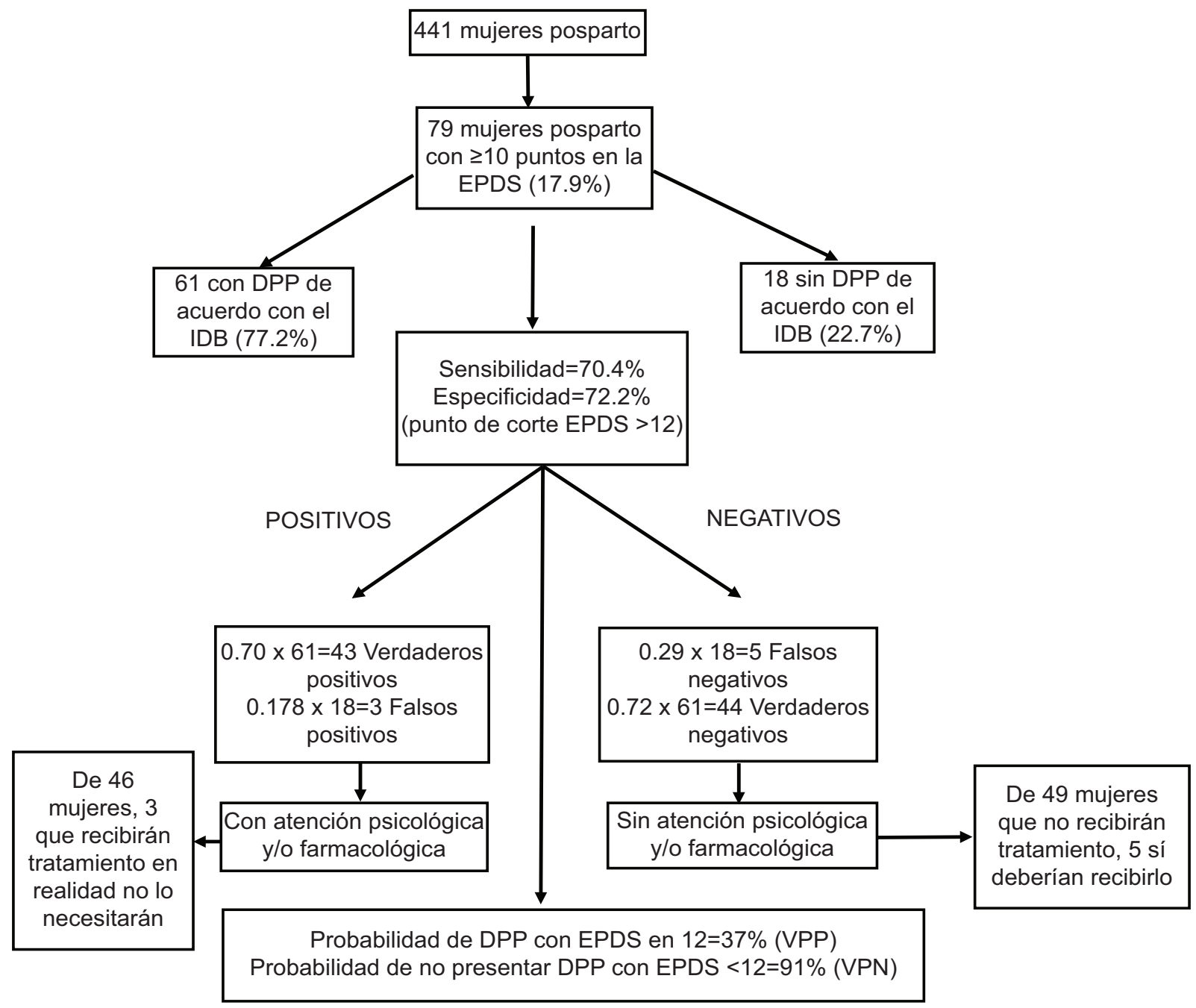

Figura 1. Consecuencias de realizar el tamizaje con la Escala de Depresión Posnatal de Edimburgo a mujeres durante el periodo posparto. $E P D S=$ Escala de Depresión Posnatal de Edimburgo, DPP = depresión posparto, IDB = Inventario de Depresión de Beck, VPP $=$ valor predictivo positivo, VPN = valor predictivo negativo. Adaptado de Leeflang MMG. Systematic reviews and meta-analyses of diagnostic test accuracy. Clin Microbiol Infect. 2014;20:105-113.

desarrollo emocional de las familias; específicamente, la atención oportuna de la DPP debe ser un aspecto prioritario.

Es importante señalar que de las mujeres con EPDS positiva, $22.7 \%$ no presentó DPP conforme a los criterios del IDB, es decir, el resultado fue falso positivo; aunado a ello, $22.8 \%$ de las mujeres que tuvieron una puntuación $\geq 10$ en la EPDS no presentaron depresión. El mayor porcentaje, $39.2 \%$, tuvo depresión leve a moderada, lo cual resulta relevante porque la identificación de la depresión en esta etapa permitirá la intervención temprana para evitar el aumento de la severidad y la cronicidad del trastorno.

El diagnóstico es un proceso dinámico cuya finalidad es apoyar en la toma de decisiones correctas.
Si bien no es preciso tener una seguridad diagnóstica absoluta para adoptar la decisión terapéutica correcta, ${ }^{23,24}$ mediante las pruebas diagnósticas se busca disminuir la incertidumbre. En este estudio, la probabilidad posprueba (valor predictivo) disminuyó la incertidumbre, ya que la probabilidad de DPP aumentó en los resultados positivos o disminuyó en los negativos al aplicar la EPDS. Dado que la probabilidad preprueba (prevalencia) de la DPP (18.7\%) no es tan alta o cercana a $100 \%$ para pasar el umbral terapéutico e iniciar tratamiento, o tan baja (0 \%) como para pasar el umbral diagnóstico y descartar DPP, se requiere aplicar una prueba sencilla, accesible y precisa. De ahí la importancia del tamizaje con la EPDS, dado que estos umbrales 


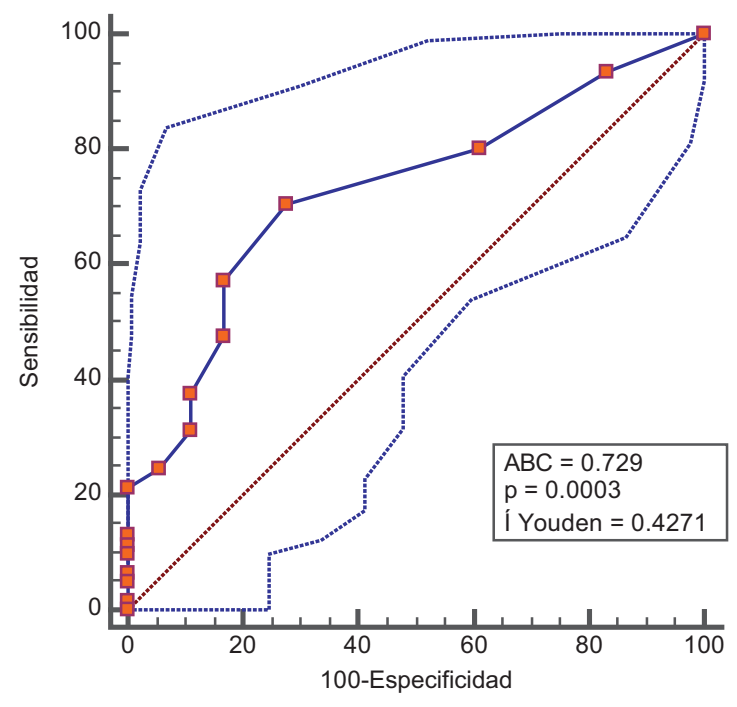

Figura 2. Curva ROC de la Escala de Depresión Posnatal de Edimburgo en la detección de depresión posparto en mujeres mexicanas. $A B C$ = área bajo la curva, Í Youden = índice de Youden.

dependen de cada escenario clínico, de la gravedad de la DPP y del costo de los casos falsos positivos y falsos negativos, tanto en términos clínicos (riesgo de retrasar o no realizar el diagnóstico, con consecuencias en la madre, la familia y el desarrollo del hijo, debido a la depresión subdiagnosticada) y económicos (si no se diagnostica, incapacidad de la madre para trabajar o gastos por el descuido del hijo debido a la depresión de la madre).

El presente estudio contribuye a conocer la validez y exactitud de la EPDS, mediante el conocimiento de la concordancia que tuvo con el IDB para clasificar correctamente la presencia o ausencia de DPP. Es sabido que cuanto más altos sean los valores de sensibilidad y especificidad, más válida será la prueba. Con un punto de corte de 12 se obtuvieron los mejores valores de sensibilidad (70.4\%) y especificidad (72.2 \%) de la EPDS, datos que reflejan el valor intrínseco de la prueba antes de conocer el resultado.

Poco se ha publicado sobre la EPDS en México. Alvarado et al. reportaron sensibilidad similar (75\%), pero mayor especificidad (93\%) con un punto de corte de $11 / 12:{ }^{25}$ el VPP (36.9\%) en nuestro estudio fue menor que el señalado por Alvarado et al. (50\%) y el VPN, similar (91.4\%). Es importante recordar que los valores predictivos dependen de la prevalencia de la DPP (18.7\%) y, por lo tanto, no son directamente aplicables a escenarios con probabilidades preprueba distintas; respecto a la DPP, los reportes difieren dependiendo del tipo de estudio, lugar de realización o instrumento utilizado como método de referencia. Por lo anterior, para conocer la probabilidad de la DPP (posprueba) en escenarios con probabilidades preprueba distintas, se calcularon las RV, que constituyen índices resumen de la sensibilidad y especificidad, independientes de la probabilidad preprueba. De tal manera, en nuestro estudio la probabilidad de encontrar un resultado positivo fue 2.5 veces mayor, lo que constituyó un cambio pequeño, aunque importante por las repercusiones psicológicas, económicas y sociales que tiene la DPP. También hubo un cambio pequeño (0.4) en la probabilidad de encontrar un resultado negativo en ausencia de DPP después de aplicar la EPDS.

El presente estudio tiene la fortaleza de que el análisis va más allá del conocimiento de los valores de la sensibilidad, especificidad y valores predictivos de la EPDS. Hasta donde conocemos, y tomando en cuenta que el proceso diagnóstico no termina con el cálculo de la probabilidad posprueba, en ningún estudio previo en México se han discutido las consecuencias del tamizaje con la EPDS. Más aún, la rentabilidad de una prueba diagnóstica no solo depende de la validez, sino también de su rendimiento en la toma de decisiones diagnóstico-terapéuticas y de su costo. ${ }^{23}$ El resultado de la EPDS sí modifica la actitud clínica inicial e influye en el manejo de las mujeres con DPP; además, la aplicación de la EPDS es sencilla, económica y accesible en la práctica clínica rutinaria, lo que no compromete su rentabilidad. En el rendimiento de una prueba diagnóstica también se deben considerar los beneficios que experimentarán las mujeres con su aplicación: mejor vínculo con el hijo, mejor desarrollo físico y emocional de los hijos, más armonía familiar y reincorporación de la mujer al trabajo, entre otros.

Una mujer con puntuación > 12 en la EPDS debe ser valorada $y$, de ser necesario, tratada oportunamente. De acuerdo con los resultados del presente estudio, se observó que de 49 mujeres consideradas sanas (y que por lo tanto no recibían tratamiento), cinco sí deberían ser derivadas para atención de la DPP; esto significa que $10 \%$ estaría sin tratamiento a pesar de la prueba de tamizaje.

Finalmente, la visualización de la curva ROC y el área bajo la curva $(0.729$, IC $95 \%=0.617-0.823)$ indica que, aun cuando es estadísticamente significativa, la EPDS tiene una exactitud moderada; por lo 
tanto, será mejor complementarla con otro instrumento o la valoración psiquiátrica de los casos sospechosos, para tener un diagnóstico más certero.

\section{Conclusión}

Es importante concientizar al personal de salud y a la población en general sobre la relevancia de la DPP, así como sobre la realización rutinaria del tamizaje para DPP con la EPDS a las madres durante las visitas de valoración del recién nacido. Cuando alguna presente > 12 puntos, se necesitará una valoración adicional con algún otro instrumento $y$, en ocasiones, enviar a la mujer al especialista, quien determinará el tratamiento. Los resultados de la EPDS son positivos en $70 \%$ de las mujeres con DPP y negativos en $72 \%$ de aquellas sin DPP. Una mujer con un resultado positivo en la EPDS tiene $37 \%$ de probabilidad de tener DPP; si su resultado es negativo, tiene $91 \%$ de probabilidad de no tener DPP. Por lo tanto, podemos afirmar que la EPDS es una prueba diagnóstica con una exactitud moderada.

\section{Conflicto de intereses}

Ninguno.

\section{Responsabilidades éticas}

Los autores declaran que para esta investigación no se realizaron experimentos en seres humanos ni en animales.

Confidencialidad de los datos. Los autores declaran que siguieron los protocolos de su centro de trabajo sobre la publicación de datos de terceros.

Derecho a la privacidad y consentimiento informado. Los autores obtuvieron el consentimiento informado de los pacientes referidos en el artículo.

\section{Bibliografía}

1. Scottish Intercollegiate Guidelines Network. Management of postnatal depression and puerperal psychosis. A National Clinical Guideline number 60. Escocia: Scottish Intercollegiate Guidelines Network; 2002.

2. Steiner M. Postpartum psychiatric disorders. Can J psychiatry. 1990;35:89-95.

3. Wisner KL, Wheeler SB. Prevention of recurrent postpartum major depression. Hosp Community Psychiatry. 1994;45:1191-1196.

4. Steiner M. Perinatal mood disorders: position paper. Psychopharmacol Bull. 1998;34:301-306

5. García L, Ortega-Soto H, Ontiveros M, Cortés J. Incidencia de la depresión posparto. Tercer Congreso Nacional de Investigación en Salud Pública. México: 1992.

6. Cox J, Murray D, Chapman G. A controlled study of the onset duration and prevalence of postnatal depression. Br J Psychiatry. 1987;163:27-31.

7. Weissman MM, Feder A, Pilowsky DJ, Olfson M, Fuentes M, Blanco C, et al. Depressed mothers coming to primary care: maternal reports of problems with their children. J Affect Disorders. 2004;78:93-100.

8. Field T. Infants of depressed mothers. Infant Behav Dev. 199;18:1-13

9. Abrams SM, Field T, Scafidi F, Prodromidis M. Newborns of depressed mothers. Infant Mental Health J. 1995;16:233-239.

10. Murray L. The impact of postnatal depression on infant development. J Child Psychol Psychiatry. 1992;33:543-561.

11. Place JMS, Billings DL, Frongillo EA, Blake ChE, Mann JR, de Castro F. Policy for promotion of women's mental health: insight from analysis of policy on postnatal depression in Mexico. Adm Policy Ment Health. 2016;43:189-198.

12. Georgiopoulos AM, Bryan TL, Wollan P, Yawn BP. Routine screening for postpartum depression. J Fam Pract. 2001;50:117-122.

13. Evins GG, Theofrasstous JP, Galvin SL. Postpartum depression: a comparison of screening and routine clinical evaluation. Am J Obstet Gynecol. 2000;182:1080-1082.

14. Hearn G, Iliff A, Jones I, Kirby A, Ormiston P, Parr P, et al. Postnatal depression in the community. Br J Gen Pract. 1998;48:1064-1066.

15. Boyd RC, Le HN, Somberg R. Review of screening instruments for postpartum depression. Arch Women Ment Health. 2005;8:141-153.

16. Cox JL, Holden JM, Sagovsky R. Detection of postnatal depression: development of the 10-item Edinburgh postnatal depression scale. $\mathrm{Br} \mathrm{J}$ Psychiatry. 1987;150:782-786.

17. Gibson J, McKenzie-McHarg K, Shakespeare J, Price J, Gray R. A systematic review of studies validating the Edinburgh Postnatal Depression Scale in antepartum and postpartum women. Acta Psychiatr Scand. 2009;119:350-364.

18. Alvarado-Esquivel C, Sifuentes-Álvarez A, Salas-Martínez C, Martínez-García S. Validation of the Edinburgh postpartum depression scale in a population of puerperal women in Mexico. Clin Pract Epidemiol Ment Health. 2006;2:33.

19. Fletcher RH, Fletcher SW, Wagner EH. Epidemiología clínica. Francia: Elsevier Masson; 1998.

20. Straus SE, Richardson WS, Glasziou P, Haynes BR. Medicina basada en la evidencia. España: Elsevier; 2006

21. Swets JA. Measuring the accuracy of diagnostic systems. Science. 1988;240:1285-1293.

22. Instituto Nacional de Estadística y Geografía. Encuesta Nacional sobre la Dinámica de las Relaciones en los Hogares 2016 (ENDIREH). Tabulados básicos. México: INEGI; 2016.

23. Ochoa-Sangrador C. Aprender a entender e interpretar las pruebas diagnósticas. Herramientas y aplicaciones. En: Aúpa, editor. Curso de actualización pediatría 2015. España: Lúa Ediciones; 2015.

24. Pauker SG, Kassirer JP. The threshold approach to clinical decision making. N Engl J Med. 1980;302:1109-1117.

25. Alvarado-Esquivel C, Sifuentes-Álvarez A, Salas-Martínez C, Martínez-García S. Clinical practice and epidemiology in mental health. Bentham Open; 2006. 